\title{
Análisis de confiabilidad en un modelo de descarga de silos de almacenamiento mediante el Método de Elementos Discretos DEM
}

\author{
Reliability analysis in an unloading model of silo storage by means of the Discrete Element \\ Method DEM \\ Fecha de entrega: 14 diciembre2013 \\ Fecha de aceptación: 9 de abril 2014
}

\section{Gerardo Arévalo-Mendoza ${ }^{1,}$ Alfonso Mariano Ramos-Cañón ${ }^{2}$ y Luis Felipe Prada-Sarmiento ${ }^{1}$}

\author{
${ }^{1}$ Departamento de Ingeniería Civil, Pontificia Universidad Javeriana, Carrera 7 № 40-62. Bogotá D.C, Colombia. \\ g.arevalo@javeriana.edu.co, lf.pradas@javeriana.edu.co \\ 2 Instituto Geofísico, Pontificia Universidad Javeriana, Carrera 7 N 42-27. Bogotá D.C, Colombia. a-ramos@javeriana.edu.co
}

La modelación con el método de elementos discretos DEM ha abierto la posibilidad de simular diversos procesos cuya naturaleza granular no se había podido tener en cuenta bajo el paradigma de la mecánica del medio continuo. La ingeniería agrícola que requiere almacenamiento de granos, la ingeniería geotécnica que requiere avanzar en el entendimiento de los procesos geomecánicos, la industria minera que requiere la optimización de diseños de taludes de corte y la industria farmacéutica, entre otros, han sido beneficiarios de los avances en la modelación de materiales granulares con el método de los elementos discretos. Sin embargo, por su naturaleza, el método de elementos discretos tiene incertidumbre del tipo epistémico y aleatorio. La incertidumbre del tipo aleatorio en la modelación con DEM se debe, entre otros, a los modelos constitutivos. Los avances reportados en la literatura muestran pocos intentos de abordar el problema de evaluación de incertidumbre en la modelación con elementos discretos, condición diferente a lo observado en modelación con elementos finitos (elementos finitos estocásticos), cuyos primeros trabajos datan de la década de los ochenta. Con el objeto de afrontar la citada deficiencia, el presente trabajo propone utilizar el procedimiento de evaluación de confiabilidad (FOSM-First Order Second Moment), que fue derivado para funciones teóricas explícitas, para la determinación de la incertidumbre aleatoria en la modelación con elementos discretos en silos de almacenamiento de granos de maíz. El método FOSM utiliza las series de Taylor para aproximar los momentos estadísticos de la función de desempeño (velocidad de descarga) con ayuda de diferencias finitas centrales. Los resultados muestran la viabilidad de usar el procedimiento para determinar la probabilidad de funcionamiento correcto de un silo para diferentes configuraciones geométricas.

Palabras clave: método de elementos discretos DEM, confiabilidad, silo, FOSM
Modelling based upon the discrete element method has opened the possibility of simulating different processes, which under the paradigm of traditional continuum mechanics, had not been duly modelled due to their granular nature. Many fields have benefited from the headway made in recent years in the modelling of granular materials with discrete elements: agricultural engineering that requires to design grain storages; geotechnical engineering that needs to deepen the understanding of geomechanical processes; the mining industry which needs to optimise the design of cut slopes for open pits; and the pharmaceutic industry among others. Despite the recent developments in the discrete element modelling, the method itself has associated an epistemic and a random uncertainty. In this case, the random uncertainty within DEM can be linked to the constitutive models. The review of specialised literature reveals few attempts to address the issue of assessing the uncertainty within simulations using DEM. On the other hand, many works have been published since the 80's about uncertainty within finite element modelling (finite element stochastics). This article presents a first approach to assess uncertainty in DEM modelling. A procedure to assess the random uncertainty in the DEM simulation of silos storing corn is proposed. FOSM (First Order Second Moment) method is used to approximate the statistical moments of the performance function (velocity of discharge), using the central finite difference technique. The obtained results allow to foresee the feasibility of using the proposed procedure to assess the probability of a non-clogged operation of the hopper system under different hopper geometries.

Keywords: discrete element method DEM, reliability, silo, FOSM 


\section{Introducción}

La modelación numérica mediante el método de elementos discretos DEM ha abierto la posibilidad de simular diversos procesos cuya naturaleza granular no se había podido tener en cuenta en simulaciones realizadas bajo el paradigma de la mecánica del medio continuo. En diversos campos es necesario conocer el comportamiento del material cuya naturaleza es discreta. Campos como la ingeniería civil que requieren dimensionar el almacenamiento y manipulación de agregados granulares en plantas de producción de concreto, o la ingeniería geotécnica que requiere avanzar en el entendimiento de los procesos geomecánicos, o la industria minera que requiere optimizar los diseños de taludes de corte de tajos abiertos, o la ingeniería agrícola y la industria farmacéutica que requieren dimensionar el correcto almacenamiento de los granos han sido algunos beneficiarios de los avances en la modelación de materiales granulares con el método de los elementos discretos. La modelación con elementos discretos DEM ha permitido el avance en el entendimiento fenomenológico de diversos procesos. En particular en los últimos años ha crecido el uso de simulaciones basadas en DEM, lo cual se ve reflejado en el creciente número de artículos publicados como lo menciona O'Sullivan (2011).

Sin embargo, como todo modelo, el método de elementos discretos posee per se incertidumbres de tipo epistémico y aleatorio. En el caso analizado en este artículo, la incertidumbre del tipo aleatorio en la modelación con DEM se atribuye a los modelos constitutivos de los contactos (partícula-partícula, partícula-pared), a pesar que en las condiciones iniciales y de contorno (condiciones geométricas y de carga) también se presenten incertidumbres de este mismo tipo. Los avances reportados en la literatura muestran contados intentos de abordar el problema de evaluación de incertidumbre en la modelación con elementos discretos como lo ha realizado Moarefvy y Verdel (2008) y Shen y Abbas (2013). Esto contrasta con las numerosas publicaciones de modelación con elementos finitos (elementos finitos estocásticos) cuyos primeros trabajos datan de la década de los ochenta (Vanmarcke y Grigoriu, 1983).

Como se mencionó anteriormente, la incertidumbre del tipo aleatorio en una simulación con elementos discretos viene dada por la falta de conocimiento de los parámetros de los modelos constitutivos que representan los contactos entre los elementos (Baecher y Christian, 2005). Una de las ventajas de la modelación con elementos discretos es la posibilidad de usar modelos constitutivos sencillos para la relación fuerza-desplazamiento en el contacto entre partículas en dirección normal y tangencial. Sin embargo, la determinación de los parámetros de dichos modelos no es una tarea que se haga con frecuencia (Fernández Llana, 2010), por lo que se observa comúnmente que los parámetros son tomados de investigaciones publicadas anteriormente, las cuales normalmente no recurren a técnicas de laboratorio para la estimación de los parámetros de los modelos constitutivos micromecánicos (Lobo-Guerrero y Vallejo, 2005; Jiang et al., 2007).

En el ámbito de la modelación macromecánica, el análisis de confiabilidad es un desarrollo que ha sido abordado desde diferentes ópticas con herramientas tales como elementos finitos (Vanmarcke y Grigoriu, 1983; Mendoza et al., 2011) y con soluciones teóricas (Kwak et al., 2010; Cherubini, 2000). Las soluciones numéricas basadas en elementos discretos para análisis de confiabilidad han tenido un menor desarrollo. Uno de los primeros trabajos en introducir un análisis probabilístico en el método de elementos discretos DEM fue el publicado por Moarefvy y Verdel (2008), en donde los resultados son derivados por medio de la modificación del código UDEC (Universal Discrete Element Code) para considerar variables de entrada del tipo probabilístico. En dicha modificación se introduce en el código el cálculo del primer y segundo momento estadístico de las variables aleatorias por medio de una aproximación a las series de Taylor. Las variables aleatorias usadas por Moarefvy y Verdel (2008) y la correspondiente función de desempeño se pueden relacionar de manera explícita bajo una ecuación analítica que vincula la resistencia al corte con el ángulo de fricción y la cohesión (criterio de falla de Coulomb).

Por otro lado, recientemente Shen y Abbas (2013) usan el método de conjuntos aleatorios cuyo objeto es proporcionar un esquema de trabajo general para tratar la información basada en conjuntos y distribuciones de probabilidad discretas. Dicha propuesta se implementó para realizar análisis de confiabilidad para estabilidad de taludes en roca.

En este trabajo se explora la potencialidad del método 
FOSM (First Order Second Moment) para determinar el primer y segundo momento estadístico de una función de variables aleatorias (Baecher y Christian, 2005) con base en el mismo andamiaje teórico empleado por Moarefvy y Verdel (2008), pero con la dificultad de no tener una función de desempeño explícita en términos de las variables aleatorias del problema (parámetros del modelo constitutivo partícula-partícula). En particular, la función de desempeño de las variables aleatorias propuesta en esta investigación será la velocidad mínima de descarga en un silo de almacenamiento de maíz. El objetivo de este artículo es determinar la probabilidad de ocurrencia de atascamiento en el punto de descarga de un silo con base en simulaciones con elementos discretos, empleando el método FOSM propuesto para resolver funciones de variables aleatorias que no tienen una ecuación explícita de relación entre ellas.

El contenido del artículo es el siguiente: primero se explica brevemente el método de elementos discretos. Después se detallan las condiciones iniciales y de contorno, así como los parámetros del modelo constitutivo junto con la incertidumbre asociada a cada parámetro del modelo. Se define la función de desempeño de las variables aleatorias a la que se le estimarán los momentos estadísticos con la metodología FOSM, para así poder calcular las probabilidades de ocurrencia de atascamiento en la descarga del silo. Se explica el método de FOSM, para finalmente presentar los resultados y las conclusiones del trabajo.

\section{Método de elementos discretos}

El método de elementos discretos DEM desarrollado por Cundall y Strack (1979) describe el comportamiento mecánico de discos en 2D o esferas en 3D. Algunos autores distinguen el DEM como un modelo lagrangiano ya que se realiza el seguimiento del movimiento de cada partícula y como tal el método establece que para un arreglo de partículas se debe verificar para cada partícula si existen contactos con las partículas vecinas y se establece una superposición entre ellas $(\delta)$. De modo que dicho "traslapo" genera una fuerza de repulsión. La fuerza de contacto resultante es descompuesta en dos partes, una normal y otra tangencial al punto de contacto. Ambas son tratadas de forma independiente, es decir, el modo como se desarrollan las fuerzas normales no afecta el desarrollo de las fuerzas tangenciales (Johnson, 1987).

La fuerza resultante (sea tangencial o normal) se relaciona con los desplazamientos mediante un modelo constitutivo o modelo de contacto. Los modelos de contacto son generalmente combinaciones de resortes (fuerza elástica), amortiguadores (fuerza viscosa) y deslizadores (fuerza de fricción), ver Figura 1. Los deslizadores se pueden encontrar en ambas direcciones (normal y tangencial). En la dirección normal se controla la fuerza máxima a tensión, mientras que en la dirección tangencial se controla el instante en el que una partícula se desliza sobre otra (O’Sullivan, 2011). Posteriormente, se aplica la segunda ley de Newton para determinar la aceleración generada por las fuerzas externas. En el método de elementos discretos se establece un tiempo de integración numérica que contempla los siguientes procedimientos durante el ciclo:

- El cálculo de la posición del elemento, integrando dos veces la aceleración (producto de la aplicación de la segunda ley de Newton) con respecto al tiempo.

- La búsqueda de contactos entre partículas.

- El cálculo de fuerzas mediante leyes constitutivas.

- El movimiento generado por la fuerza.

Al finalizar el ciclo, se actualiza la posición de la partícula en el tiempo $t+\Delta t$. Las principales suposiciones del método de elementos discretos basadas en la teoría de Hertz de la mecánica de contactos son:

- Los cuerpos son tratados como partículas rígidas, es decir las partículas mantienen su geometría durante el contacto (Johnson, 1987).

- Los contactos ocurren sobre un área pequeña.

- Se permite la superposición de las partículas rígidas si se usa la aproximación del contacto suave (Itasca, 2007). Esta aproximación es usada para simular la deformación del contacto (O’Sullivan, 2011).

El modelo constitutivo seleccionado es el simplificado de Hertz-Mindlin (Figura 1), que consta de tres partes:

1. Un modelo de rigidez de comportamiento no-lineal en la dirección normal y tangencial $\left(k_{n}\right.$ descrito en la ecuación (1), $k_{s}$ descrito en la ecuación (2)). 
2. Un amortiguamiento en ambas direcciones $\left(\xi_{n}, \xi_{s}\right)$ que provee fuerzas que son proporcionales a la diferencia de la velocidad relativa entre dos elementos en contacto (Itasca, 2007).

3. Un control deslizante de fricción en la dirección tangencial que obedece al criterio de fluencia de Coulomb $F_{y} \leq \mu_{p} F_{n}$.

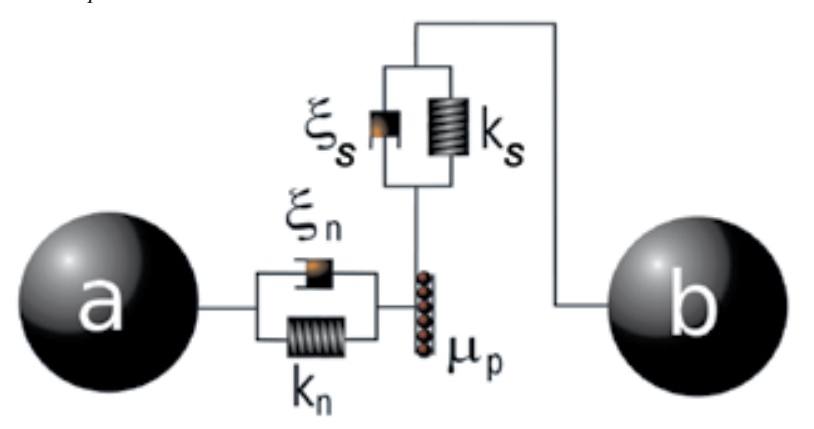

Figura 1: Modelo de contacto de Hertz-Mindlin

$$
\begin{gathered}
k_{n}=\frac{2 \bar{G} \sqrt{2 \bar{R}}}{3(1-\bar{\nu})} \sqrt{\delta} \\
k_{s}=\frac{2\left(3 \bar{G}^{2}(1-\bar{\nu}) \bar{R}\right)^{\frac{1}{3}}}{2-\bar{\nu}}\left|F_{n_{i}}\right|^{\frac{1}{3}}
\end{gathered}
$$

con,

$$
\begin{aligned}
\bar{R} & =\frac{2 R_{a} R_{b}}{R_{a}+R_{b}} \\
\bar{G} & =\frac{G_{a}+G_{b}}{2} \\
\bar{\nu} & =\frac{\nu_{a}+\nu_{b}}{2}
\end{aligned}
$$

donde para una esfera $\sqcup_{i}$ se requiere conocer el módulo de corte $G_{i}$, el coeficiente de Poisson $\nu_{i}$, el radio geométrico $R_{i}$, la fuerza normal en el contacto $F_{n_{i}}$ y el traslapo entre esferas $\delta$. Los subíndices $\sqcup_{a} \mathrm{y} \sqcup_{b}$ denotan las esferas que están en contacto, tal como se representa en el esquema de la Figura 1.

La relación de amortiguamiento se asocia con el coeficiente de restitución partícula-partícula $\alpha$ por medio de (6), que se deriva de la solución analítica de la ecuación de movimiento libre para vibración con amortiguamiento viscoso.

$$
\xi=\frac{\ln \alpha}{\sqrt{\ln ^{2}(\alpha)+\pi^{2}}}
$$

Los parámetros requeridos para el modelo de contacto son la relación de Poisson $v$, módulo de Young $E$ (o módulo de corte: $G=E /(2(1+v))$, el coeficiente de restitución partícula-partícula y el coeficiente de fricción estática partícula-muro y partícula-partícula $\left(\mu_{w}, \mu_{p}\right.$ respectivamente).

\section{Condiciones de contorno del silo y parámetros del modelo constitutivo}

La configuración geométrica del silo se presenta en la Figura 2. Se realizaron las simulaciones en el programa PFC (Itasca, 2007) con un ángulo fijo de inclinación de las paredes del silo $\vartheta$ de $45^{\circ}$. El material granular se representó como un arreglo monodisperso de aproximadamente 3000 esferas con igual diámetro $d=5 \mathrm{~mm}$. La abertura de salida del silo $D$ es variable para cada simulación. Estas aberturas de salida se definen como una relación en función del diámetro uniforme de la muestra $\left(d_{\text {gran }}=5 \mathrm{~mm}\right)$. Las relaciones de $D / d_{\text {gran }}$ usadas en la simulaciones son de 6, 8 y 10. Las partículas se generan de forma aleatoria por una única vez para cada configuración geométrica del silo, es decir, para cada abertura de la boca de descarga $D$, tratando de reducir la influencia de la variabilidad espacial en los resultados. Se genera adicionalmente una fase de estabilización en donde las partículas alcanzan el equilibrio para posteriormente quitar la abertura de la boca del silo y permitir que las partículas empiecen a fluir.

En cuanto a los parámetros del modelo constitutivo de Hertz-Mindlin, FernándezLlana (2010) propone el primer y el segundo momento estadístico de variables que se pueden considerar aleatorias para granos de maíz, información útil para la realización de un análisis de confiabilidad. Los valores usados en la modelación son: la rigidez del muro es de $10 \mathrm{MN} / \mathrm{m}$ en dirección normal y transversal $\left(k_{n}, k_{s}\right)$, el valor del coeficiente de fricción estática del muro $\mu_{w}$ es de 0.184 y la densidad de las partículas es de $1163 \mathrm{~kg} / \mathrm{m}^{3}$ (Fernández Llana, 2010). Los restantes parámetros se muestran en la Tabla 1. Se incluye además la incertidumbre aleatoria de los parámetros de los granos de maíz, expresados en términos de los estadísticos de tendencia central y de dispersión (media y el coeficiente de variación $\mathrm{CoV})$.

Los valores del coeficiente de variación $\mathrm{CoV}$, del coeficiente de fricción de las partículas y de la relación de Poisson se suponen del $50 \%$ dado que no se encontró en la 
literatura técnica datos experimentales donde se reportaran dichos valores. Coeficientes de variación del 50\% para una variable mecánica indica una alta incertidumbre si se compara con valores típicos, normalmente menores al 30\% (Baecher y Christian, 2005). Sin embargo, físicamente es admisible tener relaciones de Poisson entre $0.11 \leq v \leq 0.33$, o coeficientes de fricción entre $0.09 \leq \mu_{p} \leq 0.22$ que son los límites inferior y superior respectivamente para una función de distribución normal donde se encontraría el $64.2 \%$ de los datos en un muestreo aleatorio.

Tabla 1: Parámetros del modelo constitutivo de Hertz-Mindlin para maíz y la incertidumbre aleatoria representada en el coeficiente de variación $\mathrm{CoV}$

\begin{tabular}{|c|c|c|c|}
\hline Parámetro & Media & $\mathrm{CoV}, \%$ & Fuente \\
\hline$E, \mathrm{MPa}$ & 298 & 24 & $\begin{array}{l}\text { Fernández Llana } \\
\text { (2010) }\end{array}$ \\
\hline$v$ & 0.22 & 50 & - \\
\hline$\mu_{p}$ & 0.15 & 50 & - \\
\hline$\alpha$ & 0.748 & 4.4 & $\begin{array}{l}\text { Fernández Llana } \\
\text { (2010) }\end{array}$ \\
\hline
\end{tabular}

Para el cálculo de la velocidad de las partículas de maíz a la salida de la tobera del silo es necesario determinar un volumen de control $V C$. El volumen de control se toma desde el punto de descarga hasta $H / 2$ de altura con ancho $D$, tal como se indica en la Figura 2.

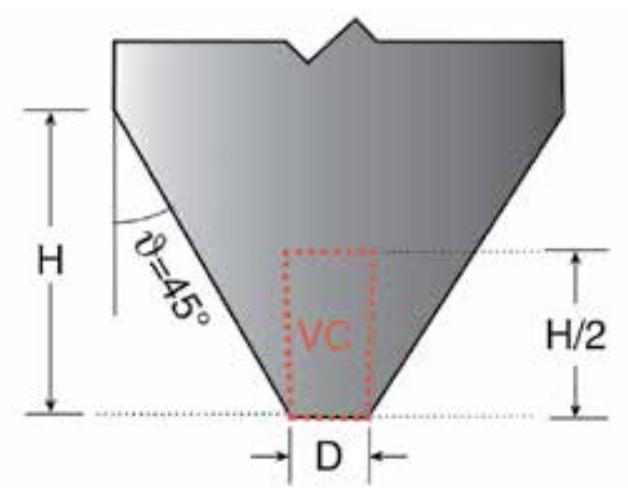

Figura 2: Esquema geométrico del silo de almacenamiento y la tobera de descarga

El volumen de control se selecciona de forma tal que se pueda evaluar la velocidad en una geometría fija que incluya tanto las partículas que están a punto de salir de la tobera, así como también aquellas partículas que están en movimiento dentro del silo, tal como se observa en la Figura 3.

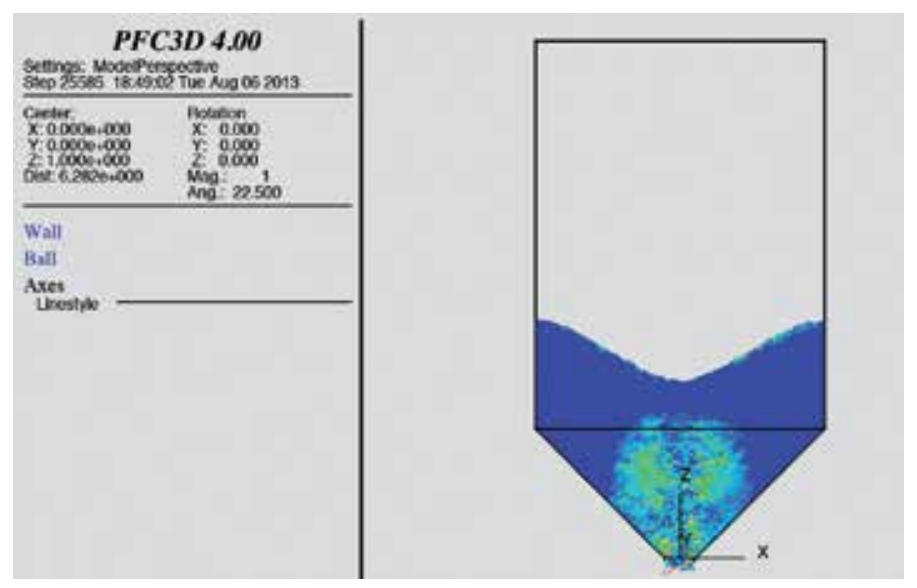

Figura 3: Imagen de la velocidad de las partículas en un instante específico de la simulación, tomada desde las funciones de imagen de PFC

\section{Método FOSM}

El método FOSM (First Order Second Moment) se utiliza para determinar los momentos estadísticos de una función de variables aleatorias. En los métodos de primer orden se usa los dos primeros términos de una expansión de la serie de Taylor (7) de la función de desempeño (velocidad de descarga $V$ ) para estimar el primer y segundo momento estadístico, es decir, el valor esperado (10) y la varianza de la velocidad (13). El método supone que la diferencia entre la realización de la variable y el valor esperado de la misma es pequeña, por lo que el cuadrado o las potencias de orden mayor son más pequeñas y se pueden ignorar (Baecher y Christian, 2005). Este método se llama de segundo momento porque la varianza tiene la forma de segundo momento y es el resultado estadístico de mayor orden usado en el análisis.

El valor esperado de la función de desempeño, en este caso la velocidad de descarga del silo $E(V)$, se obtiene a partir de la expansión de series de Taylor de la velocidad de descarga $V$ en función de las variables aleatorias $x_{i}$ que condicionan dicha velocidad: 


$$
\begin{gathered}
V\left(x_{1}, x_{2}, \ldots, x_{n}\right)=V\left(\mu_{X_{1}}, \mu_{X_{2}}, \ldots, \mu_{X_{n}}\right)+ \\
\left.\frac{1}{1 !} \sum_{i=1}^{n}\left(x_{i}-\mu_{X_{i}}\right) \frac{\partial V}{\partial x_{i}}\right|_{m}+ \\
\left.\frac{1}{2 !} \sum_{i=1}^{n} \sum_{j=1}^{n}\left(x_{i}-\mu_{X_{i}}\right)\left(x_{j}-\mu_{X_{j}}\right) \frac{\partial^{2} V}{\partial x_{i} \partial x_{j}}\right|_{m}+ \\
\frac{1}{3 !} \sum_{i=1}^{n} \sum_{j=1}^{n} \sum_{k=1}^{n}\left(x_{i}-\mu_{X_{i}}\right)\left(x_{j}-\mu_{X_{j}}\right) \\
\left.\left(x_{k}-\mu_{X_{k}}\right) \frac{\partial^{3} V}{\partial x_{i} \partial x_{j} \partial x_{k}}\right|_{m}+. .
\end{gathered}
$$

Si se considera que los términos de orden mayor al segundo son aproximadamente cero y se desarrolla la aproximación de Taylor para una función con $n$ variables aleatorias se llega a:

$$
\begin{array}{r}
V\left(x_{1}, x_{2}, \ldots, x_{n}\right) \approx V\left(\mu_{X_{1}}, \mu_{X_{2}}, \ldots, \mu_{X_{n}}\right)+ \\
\left.\sum_{i=1}^{n}\left(x_{i}-\mu_{X_{i}}\right) \frac{\partial V}{\partial x_{i}}\right|_{m}
\end{array}
$$

donde $\mu_{X_{i}}$ es el valor promedio de cada una de las variables aleatorias que tienen incertidumbre en la función de desempeño y las derivadas parciales de la velocidad de descarga $V$ son evaluadas en los valores medios de las correspondientes variables aleatorias $m=\left(\mu_{X_{1}}, \mu_{X_{2}}, \mu_{X_{n}}\right)$.

Para encontrar el valor esperado de la función $\mathrm{E}(V)$ es necesario integrar el resultado de multiplicar la función de desempeño por la función de distribución de probabilidad conjunta para todos los valores $x_{i}$ desde $-\infty$ hasta $\infty$ :

$$
\begin{array}{r}
\mathrm{E}(V)=\mu_{V} \approx V\left(\mu_{X_{1}}, \mu_{X_{2}}, \ldots, \mu_{X_{n}}\right)+ \\
\sum_{i=1}^{n} \int_{-\infty}^{\infty}\left(x_{i}-\mu_{X_{i}}\right) f_{x_{1}}\left(x_{i}\right) d x_{i}
\end{array}
$$

donde cada uno de los términos a la izquierda de la sumatoria se consideran cercanos a cero $\left(x_{i}-\mu_{X_{i}}\right) \rightarrow 0$. Estos valores multiplicados por la función de la densidad de probabilidad y el diferencial $d x_{i}$ tienden en consecuencia a cero, por lo que se encuentra una expresión simplificada para el valor esperado de la función de desempeño (velocidad de descarga en el sitio):

$$
\begin{array}{r}
\mathrm{E}(V)=\mu_{V} \approx V\left(\mu_{X_{1}}, \mu_{X_{2}}, \ldots, \mu_{X_{n}}\right)+ \\
\left.\frac{1}{2} \sum_{i=1}^{n} \sum_{j=1}^{n} \frac{\partial^{2} V}{\partial x_{i} \partial x_{j}}\right|_{m} \operatorname{Cov}\left(X_{i}, X_{j}\right)
\end{array}
$$

El segundo sumando de (10) desaparece cuando las variables aleatorias no están correlacionadas, es decir, cuando $\operatorname{Cov}\left(X_{i}, X_{j}\right)=0$ :

$$
\mu_{V}=\bar{V} \approx V\left(\mu_{X_{1}}, \mu_{X_{2}}, \ldots, \mu_{X_{n}}\right)
$$

La varianza de la función de desempeño se obtiene con base en su definición general:

$\operatorname{Var}(V)=\left.\left.\sigma_{V}^{2} \approx \sum_{i=1}^{n} \sum_{j=1}^{n} \frac{\partial V}{\partial x_{i}}\right|_{m} \frac{\partial V}{\partial x_{j}}\right|_{m} \operatorname{Cov}\left(X_{i}, X_{j}\right)$

Si se supone que las variables aleatorias son independientes entre sí, la varianza de la velocidad de descarga del silo $\sigma_{V}^{2}$ se puede simplificar como:

$$
\operatorname{Var}(V)=\sigma_{V}^{2} \approx \sum_{i=1}^{n} \sigma_{x_{i}}^{2}\left(\left.\frac{\partial V}{\partial x_{i}}\right|_{m}\right)^{2}
$$

donde $\sigma_{x_{i}}^{2}$ es la varianza de cada una de las variables aleatorias $\sqcup_{i}$ mostradas en la Tabla 1 . Si hay $n$ variables aleatorias, es necesario evaluar $n$ derivadas parciales de la función de desempeño. Cuando la función de desempeño se puede expresar en forma matemática explícita, la evaluación de cada uno de los términos de la sumatoria se puede hacer de forma analítica. En caso contrario, es necesario desarrollar una aproximación numérica para evaluar las derivadas. Como la función objetivo en este caso es la velocidad de descarga en el silo simulada mediante DEM y no tiene una expresión analítica explícita, es necesario proponer una aproximación numérica que pueda ser usada en esta situación.

\section{Diferencias finitas centrales para determi- nación de derivadas parciales}

El resultado de multiplicar el cuadrado de las derivadas parciales con la varianza de cada una de las variables 
aleatorias $\sqcup_{i}$ brinda información importante sobre la contribución parcial de cada variable aleatoria a la incertidumbre de la función objetivo, en este caso la varianza de la velocidad de descarga del silo de almacenamiento. Este artículo propone evaluar el uso del método de diferencias finitas centrales para la determinación de las derivadas parciales de la varianza de la velocidad de descarga (13).

$$
\begin{array}{r}
\frac{\partial V}{\partial x_{i}} \simeq \frac{1}{2 \Delta_{i}}\left[\left(V\left(\bar{x}_{1}, \bar{x}_{2}, \bar{x}_{i}+\Delta_{i}, \ldots, \bar{x}_{n}\right)-\right.\right. \\
\left(V\left(\bar{x}_{1}, \bar{x}_{2}, \bar{x}_{i}-\Delta_{i}, \ldots, \bar{x}_{n}\right)\right]
\end{array}
$$

Para poder desarrollar las derivadas parciales de la función de desempeño con respecto a cada una de las variables aleatorias es necesario definir $\Delta_{i}$ para (14). La magnitud de $\Delta_{i}$ debe ser lo suficientemente pequeña como para que se pueda encontrar la variación de la variable dependiente en dicho intervalo. Sin embargo, el valor $\Delta_{i}$ no puede ser en extremo pequeño porque se empiezan a generar errores debido al carácter "no derivable" o "discontinuo" de la velocidad en el tiempo que entrega el método de elementos discretos. Dado lo anterior, la selección de $\Delta_{i}$ para cada variable aleatoria es un ejercicio de ensayo y error. Después de varias ejecuciones, en el presente trabajo se seleccionó $\Delta_{i}$ con base en la Tabla 1 como una fracción de la desviación estándar de cada variable aleatoria, $\Delta_{i}=\frac{1}{6} \sigma_{x_{i}}$.

Una vez se tiene el primer (10) y segundo momento estadístico (13) de la función de variables aleatorias, es necesario seleccionar una función de densidad de probabilidad para determinar la probabilidad que la velocidad sea menor o igual a cero $\mathrm{P}(V \leq 0)$, la cual se escoge como criterio para definir la probabilidad de mal funcionamiento o atascamiento del silo. Al no tener mayor información de la forma como se distribuye probabilísticamente la variable en cuanto a sus momentos estadísticos de orden superior (asimetría, curtosis), se propone usar la función de distribución de probabilidad normal en todos los casos.

\section{Resultados}

En las Figuras 4 y 5 se presenta como ejemplo particular, la evolución en el tiempo de la simulación de la descarga del silo, de la velocidad de descarga $V$ y de la evaluación de las derivadas parciales de $V$ con respecto a la variable aleatoria del módulo de elasticidad $E$.

Para evaluar numéricamente la derivada parcial (14) es necesario evaluar la función (velocidad de descarga) tanto en los valores medios de las variables aleatorias como en el valor medio más y menos $\pm \Delta_{i}$ para cada instante de tiempo (Figura 4). Con base en estas evaluaciones, es posible calcular numéricamente (14) (derivada parcial de la velocidad con respecto a cada variable aleatoria) tal como se presenta en la Figura 5 e incorporar este resultado en la estimación de la varianza de la velocidad (13).

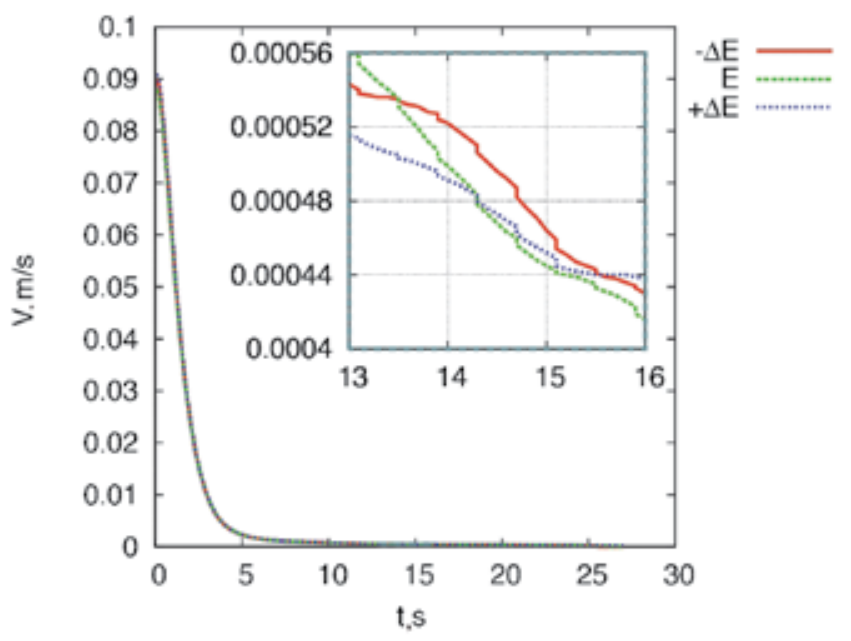

Figura 4: Evolución de la velocidad de descarga del silo para el valor medio del módulo de Young $E$, más y menos el intervalo empleado en la derivación por diferencias finitas centradas $\Delta_{H_{j}}=\frac{1}{6} \sigma_{x_{E}}$

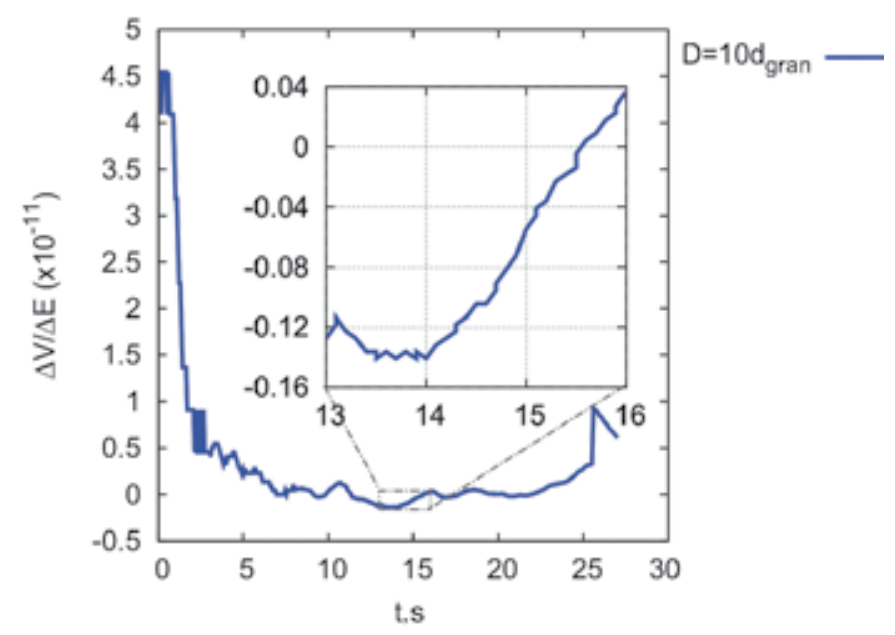

Figura 5: Evolución en el tiempo de la derivada de la velocidad de descarga con respecto al módulo de elasticidad de las esferas $E$

La Figura 6 describe el comportamiento del valor esperado de la velocidad de descarga en el volumen de control. 
Esta velocidad se obtuvo de las simulaciones DEM de los silos en donde se fijaron tres relaciones de abertura de la descarga-diámetro de partícula de $D / d_{\text {gran }}=6,8,10$. La simulación se desarrolló completamente hasta que los silos se descargaran completamente. El valor esperado de la velocidad de descarga se calculó con base en (10).

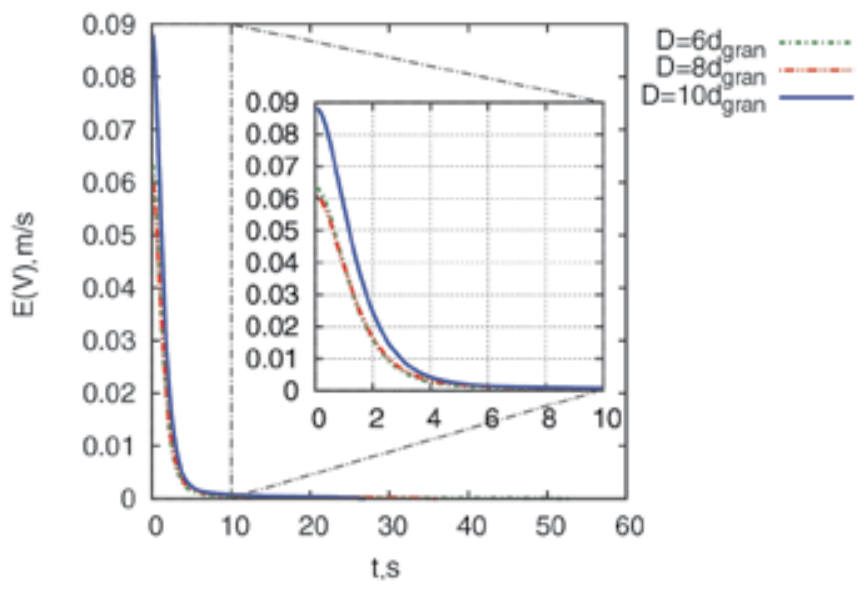

Figura 6: Evolución del valor esperado de la velocidad de descarga del silo en el tiempo $\mathrm{E}(V(t))$ para la relación de abertura de descarga - diámetro de la partícula $D / d_{\text {gran }}=6,8,10$

Adicionalmente, en la Figura 6 se hace un acercamiento para ver en detalle la evolución en el tiempo de la velocidad de descarga durante los primeros $10 \mathrm{~s}$ de descarga de los silos. Se observa que entre más grande sea la relación $D /$ $d_{\text {gran }}$ la velocidad de descarga es mayor. Sin embargo, las diferencias entre las relaciones 6 y $8 D / d_{\text {gran }}$ son menores que entre 8 y $10 D / d_{\text {gran. }}$. Es interesante notar que al inicio del proceso, la velocidad de las partículas en el volumen de control alcanza su valor más alto y a medida que pasa el tiempo la velocidad de descarga disminuye hasta llegar a cero, condición en la cual el silo se ha descargado completamente.

Por otro lado, la Figura 7 muestra la variación de la desviación estándar de la velocidad de descarga del silo en el tiempo para las tres relaciones $D / d_{\text {gran }}$. Se observa que el factor de relación ancho de la descarga - diámetro máximo de la partícula $D / d_{\text {gran }}$ influye en la incertidumbre aleatoria de la velocidad de descarga del silo. A mayor relación $D / d_{\text {gran }}$, la desviación estándar de la velocidad se hace más grande, es decir aumenta la incertidumbre en la estimación de la velocidad de descarga.

La Figura 8 presenta la evaluación del coeficiente de variación $\mathrm{CoV}$ de la velocidad de descarga de los silos,

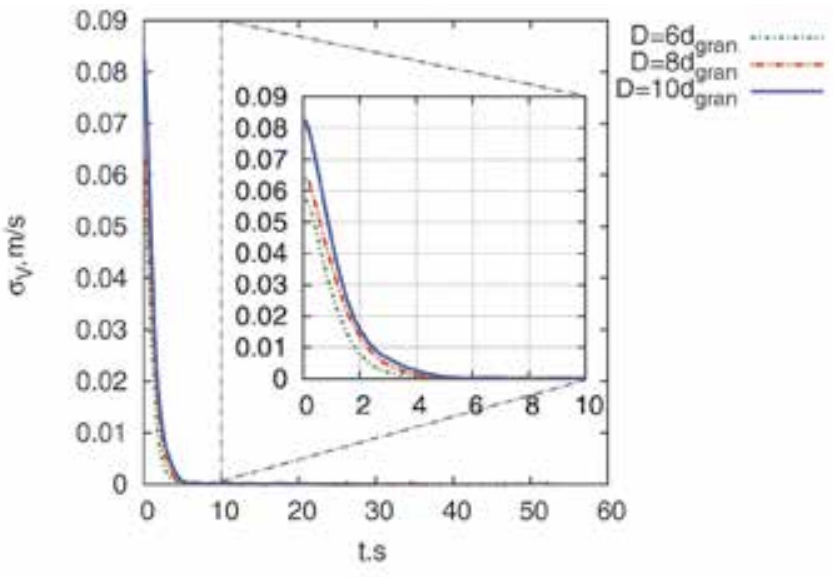

Figura 7: Evolución de la desviación estándar de la velocidad de descarga del silo en el tiempo para relación de abertura de descarga - diámetro de la partí1cula $D / d_{\text {gran }}=6,8,10$

calculada con base en los resultados de las Figuras 6 y 7 . No es clara la tendencia de la evaluación de la incertidumbre normalizada cuando se calcula el coeficiente de variación para las diferentes relaciones $D / d_{\text {gran. }}$. Por un lado se observa un aumento del valor esperado de la velocidad de descarga cuando crece la relación $D / d_{\text {gran }}$ lo que indicaría que la probabilidad de atascamiento disminuiría con el incremento de la relación $D / d_{\text {gran, }}$, suponiendo que la desviación estándar se mantenga constante. Por otro lado, la desviación estándar también se incrementa con el aumento de la abertura $D / d_{\text {gran }}$ lo que conduciría a que la probabilidad de atascamiento se incremente con el aumento de la relación abertura-diámetro, manteniendo el valor esperado constante. Esto contradeciría la tendencia mencionada anteriormente en función del análisis del valor esperado. Dado lo anterior, y tal como se ve reflejado en la Figura 8, no hay una tendencia claramente establecida de la variación del coeficiente de variación que permita inferir cuál será el comportamiento de la probabilidad de atascamiento para las diferentes relaciones de aberturasdiámetros.

La evolución de la probabilidad de tener una velocidad promedio de descarga en el volumen de control menor o igual que cero $\mathrm{P}(\bar{V}) \leq 0$ en función del tiempo para las diferentes relaciones $D / d_{\text {gran }}$ se presenta en la Figura 9. Esta probabilidad representa la condición de falla de la operación del silo, es decir, indica el momento en el cual se presentaría la condición de atascamiento en la descarga. La probabilidad asociada a las diferentes relaciones de abertura-diámetro es similar. Al inicio del proceso de 


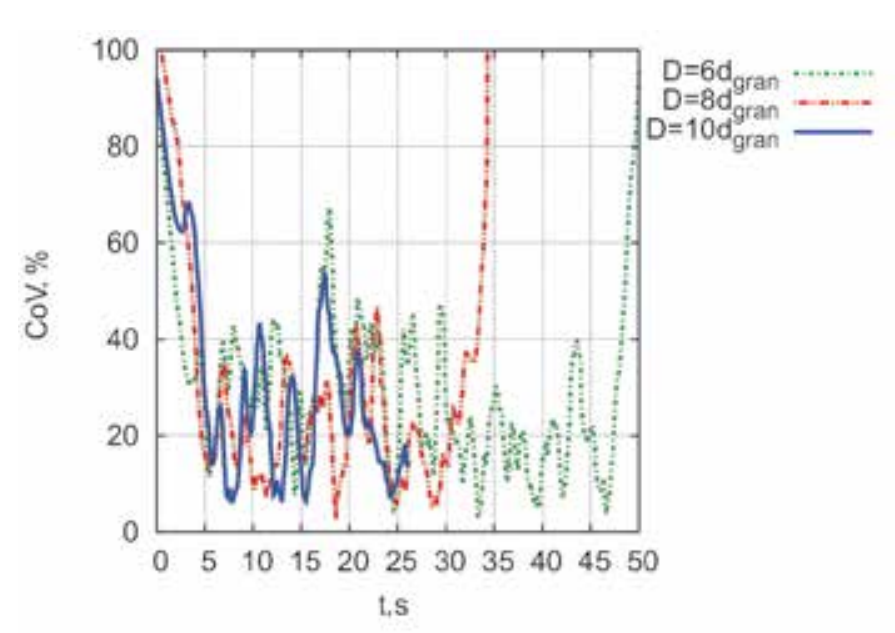

Figura 8: Evolución del coeficiente de variación de la velocidad de descarga en el tiempo

descarga, la probabilidad de atascamiento es mayor con valores entre el 12 y el $18 \%$ a pesar que el valor medio de la velocidad en los casos considerados es cercano a $1 \mathrm{~m} / \mathrm{s}$. A medida que transcurre el tiempo, la probabilidad de atascamiento disminuye rápidamente acercándose a cero, es decir el material granular fluye libremente por la descarga del silo. Eventualmente se presentan leves aumentos en la probabilidad de atascamiento producto de la no continuidad de la velocidad de descarga durante el proceso. Para tiempos aproximados de 25, 34 y $50 \mathrm{~s}$ se observa que la probabilidad de atascamiento aumenta vertiginosamente para relaciones $D / d_{\text {gran }}=6,8$, y 10 respectivamente con valores de $\mathrm{P}(\bar{V}) \approx 0.5$ (en la Figura 9 se muestra únicamente la escala vertical hasta valores de $0.2)$.

Al final del proceso de descarga del silo, se registran probabilidades de atascamiento cercanas a cero para las tres relaciones abertura-diámetro de las partículas. En realidad, esto no representa una situación de atascamiento de partículas. El aumento observado de la probabilidad de atascamiento está asociado a que la velocidad de las partículas en el volumen de control se hace cero ya que el silo se descargó completamente. Es decir, el momento en que se vacía el silo se ve mejor reflejado en la cuantificación de la probabilidad de atascamiento de la Figura 9 que en la Figura 6, aunque físicamente el silo en realidad no experimenta atascamiento en la descarga.

Es importante anotar que se deben presentar diferencias en los resultados cuando el análisis se hace en dos o tres dimensiones. La condición de fricción de las paredes es mayor que la fricción entre partícula y partícula, por lo que se espera que las probabilidades de atascamiento sean mayores en un análisis de 3 dimensiones. Sin embargo, el procedimiento propuesto en este artículo tiene la versatilidad de poderse utilizar en un análisis bidimensional o tridimensional sin necesidad de introducir cambio alguno en la formulación.

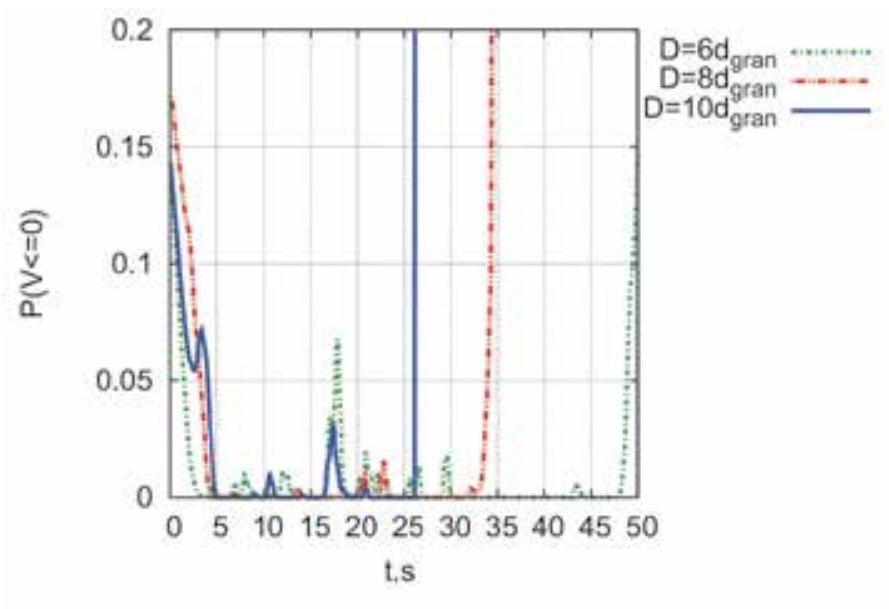

Figura 9: Evolución de la probabilidad de tener velocidad de descarga menor o igual a cero en función del tiempo

\section{Conclusiones}

Esta investigación introduce un procedimiento novedoso para la evaluación de la confiabilidad en la resolución de problemas simulados con el método de elementos discretos DEM.

Se presentó la implementación numérica de la teoría First Order Second Moment en el análisis de la probabilidad de atascamiento en silos de almacenamiento de granos, con el objeto de incorporar la incertidumbre aleatoria de los parámetros físicos que permiten modelar la interacción entre esferas que representan materiales granulares.

La metodología propuesta permite analizar la influencia de cada variable aleatoria en la incertidumbre de una función de desempeño, por medio del estudio de la evolución de la contribución de cada variable a la varianza de la función.

En el caso particular de la simulación de la descarga de un material granular almacenado en un silo, se pudo concluir que la relación entre el diámetro de la abertura y el tamaño máximo de los granos influye directamente en la velocidad de descarga, en el aumento de la incertidumbre en la 
estimación de la velocidad, así como en la probabilidad de atascamiento del sistema.

En términos generales se observa que la probabilidad de atascamiento, es decir, la probabilidad de tener velocidades menores que cero, es mayor a medida que la relación $D / d_{\text {gran }}$ es menor. Lo anterior se puede deber a que a medida que la abertura de la boca de descarga es menor, se presenta mayor interacción entre las partículas generando un posible efecto de arco. Dicha interacción se ve reflejada en el aumento en el coeficiente de variación de la velocidad de descarga que a su vez genera mayores probabilidades de atascamiento.

Se demuestra el potencial uso del procedimiento propuesto y se abre la puerta para seguir trabajando en aplicaciones para la ingeniería y la industria en donde los materiales granulares son el objeto de análisis y su naturaleza particulada se ve mejor representada con técnicas de modelación discreta.

La combinación planteada en este artículo entre métodos de análisis basados en la confiabilidad y técnicas de simulación numérica discreta permitirá tomar mejores decisiones en el futuro acerca de las condiciones mecánicas requeridas para disminuir de forma racional la probabilidad de falla de cualquier sistema geotécnico.

\section{Agradecimientos}

Los autores agradecen a la Pontificia Universidad Javeriana sede Bogotá por el apoyo económico al presente trabajo por medio de la financiación del proyecto de investigación 4709 - Inestabilidades en materiales granulares, un estudio a nivel de la microescala.

\section{Referencias}

Baecher, G. B. and Christian, J. T. (2005). Reliability and statistics in geotechnical engineering. Wiley

Cherubini, C. (2000). Probabilistic approach to the design of anchored sheet pile walls. Computers and Geotechnics 26(3):309-330.

Cundall, P. A. and Strack, O. D. L. (1979). A discrete numerical model for granular assemblies. Géotechnique 29(1):47-65.
Fernández Llana, D. (2010). Determinación de parámetros utilizados en las simulaciones DEM. Tesis de Máster, Universidad Politécnica de Madrid

Itasca (2007). Particle flow code 3D, version 4D. Itasca Consulting Group Inc., Minneapolis, MN, USA

Jiang, M., Yu, H.-S. and Leroueil, S. (2007). A simple and efficient approach to capturing bonding effect in naturally microstructured sands by discrete element method. International Journal for Numerical Methods in Engineering, 69(6):1158-1193

Johnson, K. L. (1987). Contact mechanics. Cambridge University Press.

Kwak, K., Kim, K. J., Huh, J., Lee, J. H. and Park, J. H. (2010). Reliability-based calibration of resistance factors for static bearing capacity of driven steel pipe piles. Canadian Geotechnical Journal 47(5):528-538.

Lobo-Guerrero, S. and Vallejo, L. E. (2005). Discrete element method evaluation of granular crushing under direct shear test conditions. Journal of Geotechnical and Geoenvironmental Engineering 131(10):1295-1300.

Mendoza, C., Ramos, A. M., Cunha, R. y Lizcano, A. (2011). Análisis del proceso constructivo y variabilidad de parámetros geotécnicos en la capacidad de carga de micropilotes usando elementos finitos aleatorios. Obras y Proyectos 11:61-70.

Moarefvy, P. and Verdel, T. (2008). The probabilistic distinct element method. International Journal for Numerical and Analytical Methods in Geomechanics 32(5):559-577.

O’Sullivan, C. (2011). Particulate Discrete Element Modelling: A Geomechanics Perspective. Applied Geotechnics. Spon Press/ Taylor \& Francis. Wiltshire, Great Britain.

Shen, H. and Abbas, S. M. (2013). Rock slope reliability analysis based on distinct element method and random set theory. International Journal of Rock Mechanics and Mining Sciences 61:15-22.

Vanmarcke, E. and Grigoriu, M. (1983). Stochastic finite element analysis of simple beams. Journal of Engineering Mechanics 109:1203-1214. 